\title{
The fence experiment - full-scale lidar-based shelter observations
}

\author{
Alfredo Peña, Andreas Bechmann, Davide Conti, and Nikolas Angelou \\ DTU Wind Energy, Technical University of Denmark, Roskilde, Denmark \\ Correspondence to: Alfredo Peña (aldi@dtu.dk) \\ Received: 26 March 2016 - Published in Wind Energ. Sci. Discuss.: 4 April 2016 \\ Revised: 7 July 2016 - Accepted: 8 July 2016 - Published: 14 July 2016
}

\begin{abstract}
We present shelter measurements of a fence from a field experiment in Denmark. The measurements were performed with three lidars scanning on a vertical plane downwind of the fence. Inflow conditions are based on sonic anemometer observations of a nearby mast. For fence-undisturbed conditions, the lidars' measurements agree well with those from the sonic anemometers and, at the mast position, the average inflow conditions are well described by the logarithmic profile. Seven cases are defined based on the relative wind direction to the fence, the fence porosity, and the inflow conditions. The larger the relative direction, the lower the effect of the shelter. For the case with the largest relative directions, no sheltering effect is observed in the far wake (distances $\gtrsim 6$ fence heights downwind of the fence). When comparing a near-neutral to a stable case, a stronger shelter effect is noticed. The shelter is highest below $\approx 1.46$ fence heights and can sometimes be observed at all downwind positions (up to 11 fence heights downwind). Below the fence height, the porous fence has a lower impact on the flow close to the fence compared to the solid fence. Velocity profiles in the far wake converge onto each other using the self-preserving forms from two-dimensional wake analysis.
\end{abstract}

\section{Introduction}

The flow around obstacles is difficult to observe and model because of the turbulence characteristics and velocity shears. Such flow has not received much attention in wind energy partly due to the urge to decrease the cost of energy, narrowing the research on flow characteristics to large-turbine operating conditions. These turbines generally operate in areas and at heights where the obstacles' effects can be neglected. However, due to the decrease of available "high wind" sites on land, turbines are being deployed in environments where obstacles cannot be ignored. Also, the "small" turbine industry has steadily grown (Gsänger and Pitteloud, 2014) and small machines are commonly installed close to obstacles. In Denmark, small turbines $(<25 \mathrm{~kW})$ are normally placed closer than $20 \mathrm{~m}$ from buildings and below $25 \mathrm{~m}$ from the ground. Due to shelter, such installations often result in lower-than-expected yields and turbine breakdown.

Computational fluid dynamics (CFD) methods, e.g. those solving the Reynolds-averaged Navier-Stokes (RANS) equations, can accurately describe the flow around obstacles and are used to study specific flow conditions (Iaccarino et al., 2003). However, they are often too expensive to be implemented in wind-resource assessment tools. Therefore, the obstacles' effect is normally estimated using "engineering"-like models. Some, e.g. WEMOD (Taylor and Salmon, 1993) and WAsP-shelter (Mortensen et al., 2007), are based on the analytical theory by Counihan et al. (1974), which describes the wake behind two-dimensional (2-D) obstacles, and consider wakes behind three-dimensional (3-D) objects.

Analytical theories and CFD simulations have mainly been evaluated with wind-tunnel data (Castro and Robins, 1977) and few full-scale 3-D shelter experiments have been performed. Nägeli (1953) is perhaps the first to investigate the mean velocity profiles downwind of porous windbreaks, although his data are not of the highest quality (Seginer, 1972). Most shelter experiments are associated with agroengineering studies, where the purpose is windbreak optimization for stock and crop protection, and are focused on porous obstacles (Nord, 1991). Wilson (2004) describes a field experiment where the shelter of a $1.25 \mathrm{~m}$ high and $114 \mathrm{~m}$ 
wide porous plastic fence was measured as a function of the "obliquity" of the inflow; the fence seems to have an effect on the flow for all the measurement positions (and all obliquities) along a transect that extends 20 fence heights at a vertical level about half the fence height. Shelter effects in oblique flows are studied by numerical simulations in Wang and Takle (1996). A review on modelling and simulation studies of windbreaks that includes comparison with experimental data is presented by Wang et al. (2001).

Here, we present a comprehensive dataset of full-scale measurements of a fence shelter. The measurements were conducted at Ris $\varnothing$ 's test site in Denmark and the WindScanner (WS) lidar-based system was used to measure the 3-D wind vector on a vertical plane for different inflow conditions. The experiment is somewhat similar to that of Wilson (2004) but we concentrated our measurement efforts close to the fence (both horizontally and vertically). The experiment's objective is to serve as benchmark for shelter models. Section 2 introduces the definitions and theory used to analyse the measurements. Section 3 provides details of the site and the measurements, Sect. 4 describes the way data are analysed, and Sect. 5 presents the shelter results for a number of inflow conditions or cases. Finally, Sect. 6 provides some discussion and conclusions about the campaign and future model evaluation.

\section{Definitions}

\subsection{Problem}

We describe the turbulent flow behind a 2-D fence (Fig. 1) and compare it to the undisturbed inflow (subscript ${ }_{o}$ ). We use a right-handed Cartesian coordinate system with the three velocity components, $u, v$, and $w$, aligned with the $x, y$, and $z$ (the vertical) axes, respectively. The horizontal wind-speed magnitude is thus $U=\left(u^{2}+v^{2}\right)^{1 / 2}$. The coordinate centre is placed on the ground at the fence. The flow is described by the roughness length $z_{o}$ and the fence height $h$.

We investigate the flow on a 2 -D vertical plane extending $2.5 h$ vertically and $\approx 11 h$ horizontally downstream of the fence. For simplicity, two main regions are defined in this plane: the "near-wake" $(x<6 h)$ and the "far-wake" $(x>6 h)$ regions. In the analysis below, we describe the flow for different inflow directions and that perpendicular to the fence (along the $x$ axis).

\subsection{Inflow}

We assume that the inflow can be described by the diabatic wind profile (Stull, 1988),

$U_{o}(z)=\frac{u_{*}}{\kappa}\left[\ln \left(\frac{z}{z_{o}}\right)-\psi_{m}(z / L)\right]$,

where $u_{*}$ is the friction velocity, $\kappa$ the von Kármán constant $(\approx 0.4)$, and $\psi_{m}$ a function of the dimensionless stability pa-

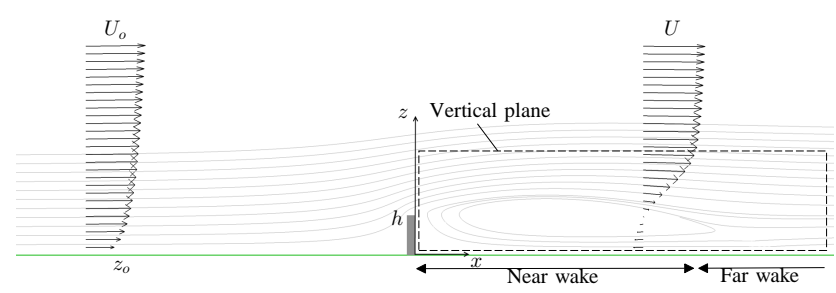

Figure 1. Simulated turbulent flow around a 2-D fence of height $h$ and the vertical plane of interest. The flow is simulated with the model described in Sect. 3.3.1.

rameter $z / L, L$ being the Obukhov length. $u_{*}$ and $L$ can be computed as

$$
\begin{aligned}
& u_{*}=\left({\overline{u^{\prime} w^{\prime}}}^{2}+{\overline{v^{\prime} w^{\prime}}}^{2}\right)^{1 / 4}, \\
& L=-\frac{u_{*}{ }^{3}}{\kappa(g / \bar{T}) \overline{w^{\prime} \Theta_{\mathrm{v}}^{\prime}}},
\end{aligned}
$$

where $g$ is the gravitational acceleration, $T$ a reference temperature, $\Theta_{v}$ the virtual potential temperature; the primes denote fluctuations around the time average, and the overbar a time average.

\subsection{Two-dimensional wake theory}

The wind-speed ratio $U / U_{o}$ at a specific height $z$ is used to quantify the shelter and can be written as

$\frac{U(x, z)}{U_{o}(z)}=1-\frac{\Delta U(x, z)}{U_{o}(h)} \frac{U_{o}(h)}{U_{o}(z)}$,

where $\Delta U(x, z)=U_{o}(z)-U(x, z)$. The term $\Delta U(x$, $z) / U_{o}(h)$ is predicted by the Counihan et al. (1974) analytical theory, in which a 2-D obstacle wake is divided into three regions. Within the mixing region, spreading from the obstacle's top, the velocity is self-preserving with the form,

$$
\begin{aligned}
\frac{\Delta U(x, z)}{U_{o}(h)}= & \frac{C / I(n)}{K h^{2} U_{o}(h)^{2}}\left(\frac{x}{h}\right)^{-1} \frac{d}{d \eta}\left[\eta _ { 1 } ^ { 2 } F _ { 1 } \left(\frac{2-n}{2+n},\right.\right. \\
& \left.\left.\frac{n+4}{2+n}, \frac{-\eta^{n+2}}{(n+2)^{2}}\right)\right],
\end{aligned}
$$

where $C$ is related to the wake strength (see below), $K=2 \kappa^{2} / \ln \left(h / z_{o}\right), n$ the inflow's shear exponent, ${ }_{1} F_{1}$ the confluent hypergeometric function, $\eta$ a dimensionless length scale related to the mixing-region depth, and $I$ an integral constant for the wake's self-preserving solution in the mixing region. The latter two are expressed as

$I(n)=\frac{(1+n)(2+n)^{(4+n) /(2+n)}}{1+2 n} \frac{\Gamma\left(\frac{4+n}{2+n}\right) \Gamma\left(\frac{1-n}{2+n}\right)}{\Gamma\left(\frac{2-n}{2+n}\right)}$, 


$$
\eta=\left(\frac{z}{h}\right)\left[\frac{K x}{h}\right]^{-1 /(n+2)}
$$

Counihan et al. (1974) show that profiles of $\frac{\Delta U(x, z)}{U_{o}(h)}\left(\frac{x}{h}\right)$ as function of $\eta$ converge onto each other within the farwake region $6 \leq x / h \leq 30$ from full-scale measurements of the wind behind porous windbreaks and within the range $7.5 \leq x / h \leq 72$ from wind-tunnel measurements. Based on Counihan et al. (1974) theory and using wind-tunnel measurements behind 2-D fences, Perera (1981) proposes an expression that has become the basis of engineering obstacle models,

$$
\frac{\Delta U(x, z)}{U_{o}(h)}=A(1-\varphi)\left(\frac{x}{h}\right)^{-1} \eta \exp \left(-0.67 \eta^{1.5}\right),
$$

where $\varphi$ is the fence porosity and $A$ a constant $(=9.75)$.

The solution to the term $\frac{d}{d \eta}[\ldots]$ in Eq. (5) is unattractive but for the special case $n=0$, it is simple $\left(=2 \eta \exp \left(-0.25 \eta^{2}\right)\right)$. The self-similar profile $\frac{\Delta U(x, z)}{U_{o}(h)}\left(\frac{x}{h}\right)$ shows a maximum at $\eta(z / h \approx 1)$ and approaches zero with increasing $\eta$. For decreasing $n$ values, the zero approach occurs at smaller $\eta$ values and the profile's maximum slightly decreases (only $7 \%$ between $n=0.14$ and 0 ). Also, $I$ is not that sensitive to $n(=7.64$ and 7.08 for $n=0.14$ and 0 , respectively). The expression $C=C_{h} h^{2} U_{o}(h)^{2}$ is derived by Counihan et al. (1974) based on pressure measurements on blocks in shear flows from the Building Research Station Digest no. 119 (1970). Therefore, Eq. (5) can be simplified to

$$
\frac{\Delta U(x, z)}{U_{o}(h)}=\frac{C_{h}}{K I(n=0.14)}\left(\frac{x}{h}\right)^{-1} 2 \eta \exp \left(-0.25 \eta^{2}\right) .
$$

Counihan et al. (1974) chose $C_{h}=0.8$ for measurements behind 2-D blocks. Following the analysis by Taylor and Salmon (1993), $C_{h}$ corresponds to the wake-moment coefficient. They suggest $C_{h}=B(1-\varphi)$ with $0.2 \leq B \leq 0.8$ depending on the obstacle type.

\section{Site and measurements}

We aim at describing the effect of a full-scale obstacle on the atmosphere by measuring on a vertical plane downwind of a fence. Here, we first describe the site, the inflow conditions from mast measurements, and the shelter measurements performed by the WS.

\subsection{Site}

The "fence experiment" took place at Ris $\varnothing$ 's test site, which is $\approx 7 \mathrm{~km}$ north from Roskilde and $\approx 35 \mathrm{~km}$ west from Copenhagen, Denmark (Fig. 2). It was conducted during two periods: from 10 March to 1 April the fence was solid and from 29 September to 2 October 2015 the fence was made

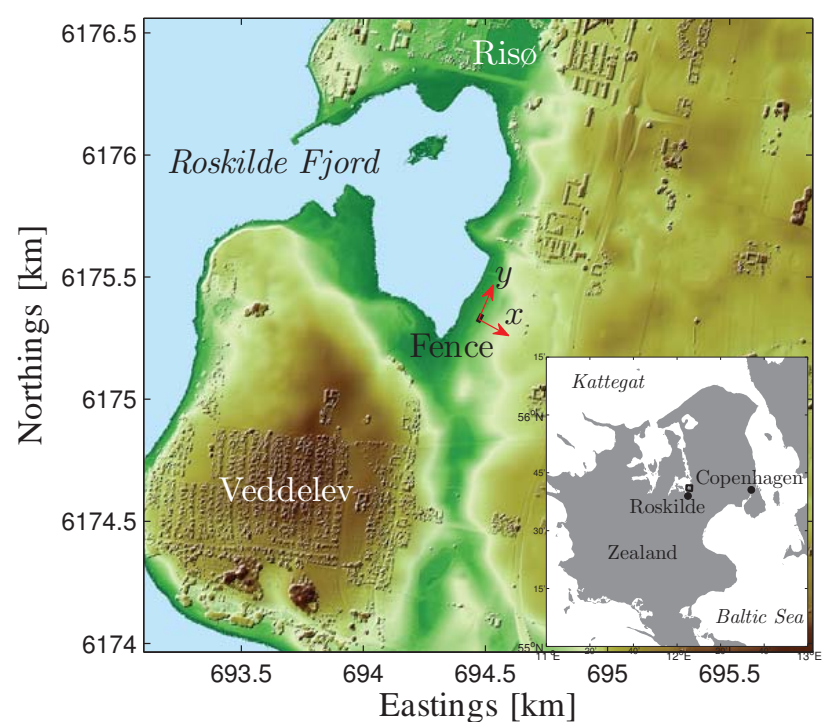

Figure 2. The fence experiment on a digital surface model (UTM32 WGS84) of the area surrounding Ris $\varnothing$ 's test station. Cropland and grassland are shown in green; cropland and artificial land in light brown, rural areas and buildings in brown, and the waters from the fjord in light blue. The reference coordinate system is shown in red. In the bottom-right part, the test site location (black rectangle) on the island of Zealand, Denmark, is illustrated.

porous. The terrain at the site is slightly hilly and the surface is characterized as a mix between cropland, grassland, artificial land, and coast.

The fence is made of horizontal wooden panels with wooden beams on each side supporting the structure (see Fig. 3, bottom panels). For the second period of the experiment, the fence porosity (ratio of the "pores" to the total area) is 0.375 . The fence is $3 \mathrm{~m}$ high, $30 \mathrm{~m}$ wide, and $0.04 \mathrm{~m}$ thick (the wooden vertical poles are $0.1 \mathrm{~m}$ thick). The centre point of the fence has coordinates $694477.5^{\circ} \mathrm{E}, 6175332^{\circ} \mathrm{N}$ (UTM32 WGS32) and is $\approx 78 \mathrm{~m}$ south-east of the Roskilde Fjord coastline. Due to land restrictions and the orientation of the coastline, the fence is oriented $\approx 42^{\circ}$ from the true north (winds from the direction $\approx 312^{\circ}$ are normal to the fence).

The terrain's slope behind the fence was measured with a Trimble global positioning system (GPS), along two lines from its corners. Figure 4 (top panel) illustrates the fence experiment and the instrumentation. Figure 4 (bottom panel) illustrates the positions where we measured on the vertical plane (described in Sect. 3.3) and the terrain elevation. Note that the reference system is not at the fence centre but $1.53 \mathrm{~m}$ south-west and so fence corners are not at the same distance from the reference system (see Table 1). The terrain height above the fence base for the positions at which we measured the shelter is provided at http://www.fence.vindenergi. dtu.dk. The relative direction to the fence, $\theta$, is defined positively increasing clockwise. 


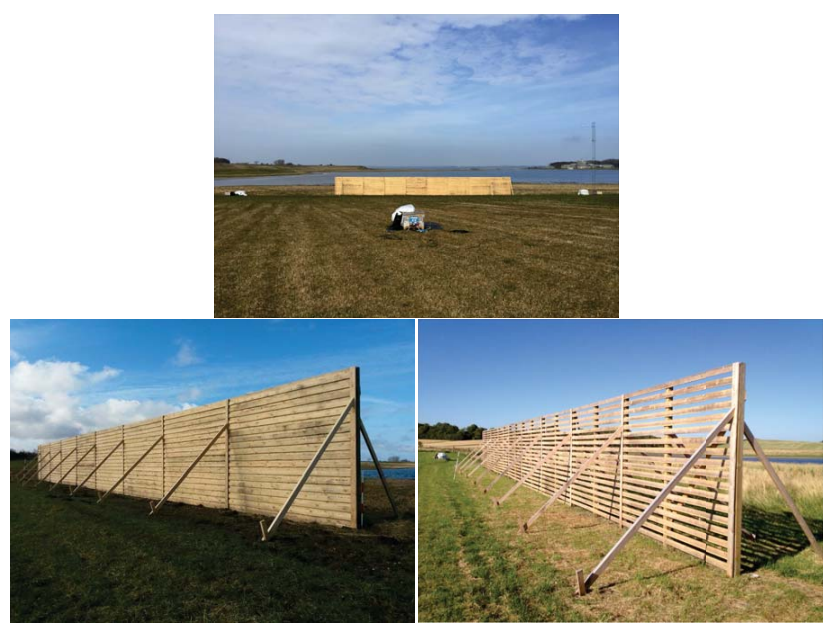

Figure 3. Photographs of the fence experiment. Top panel: overview of the fence and the instrumentation including the lidars and the mast. Left panel: solid and right panel: porous fence setups.

\subsection{Meteorological mast}

A mast was deployed north-east of the fence and two Metek USA-1 sonic anemometers were placed on booms oriented towards the fence at 6 and $12 \mathrm{~m}$ above the ground and recorded time series of the three wind-speed components and temperature at $20 \mathrm{~Hz}$. Mean and turbulence statistics are estimated over $10 \mathrm{~min}$ periods from the sonic anemometer measurements (we also analyse the sonic anemometer time series in shorter time periods as described in Sect. 4). The sonic anemometer times series are linearly detrended over the $10 \mathrm{~min}$ period, and mean and turbulence quantities like $u_{*}$ and $L$ are estimated from the $10 \mathrm{~min}$ statistics. The terms $T$ and $\overline{w^{\prime} T_{\mathrm{v}}^{\prime}}$ in Eq. (3) are estimated from the sonic anemometer measurements of temperature and kinematic heat flux, respectively. For the latter, we use the crosswind corrections of Liu et al. (2001).

\subsection{Lidar measurements}

\subsubsection{Basics}

The three velocity components on the vertical plane were measured using three short-range lidars that were synchronized both in time and space. These three devices comprise the WS. The instruments are based on a continuouswave (CW) coherent lidar (Karlsson et al., 2000), which is capable of measuring the radial (or line-of-sight) speed and its sign (Sjöholm et al., 2014). The minimum and maximum measurement ranges and absolute detectable speeds of the WS's lidars are 8 and $150 \mathrm{~m}^{1}$, and $\approx 0.15$ and $18 \mathrm{~m} \mathrm{~s}^{-1}$, respectively.

\footnotetext{
${ }^{1}$ Limitations imposed by the hardware design and size of the probe volume.
}

Table 1. Instrumentation coordinates for the fence experiment.

\begin{tabular}{llll}
\hline Instrument & $x[\mathrm{~m}]$ & $y[\mathrm{~m}]$ & $z[\mathrm{~m}]$ \\
\hline R2D1 & 2.43 & -27.67 & 0.40 \\
R2D2 & 2.36 & 26.96 & 0.40 \\
R2D3 & 43.00 & 0 & 2.43 \\
Sonic anemometer & 0.06 & 31.91 & 6,12 \\
Fence (southern corner) & 0 & -13.51 & 0,3 \\
Fence (northern corner) & 0 & 16.53 & 0,3 \\
\hline
\end{tabular}
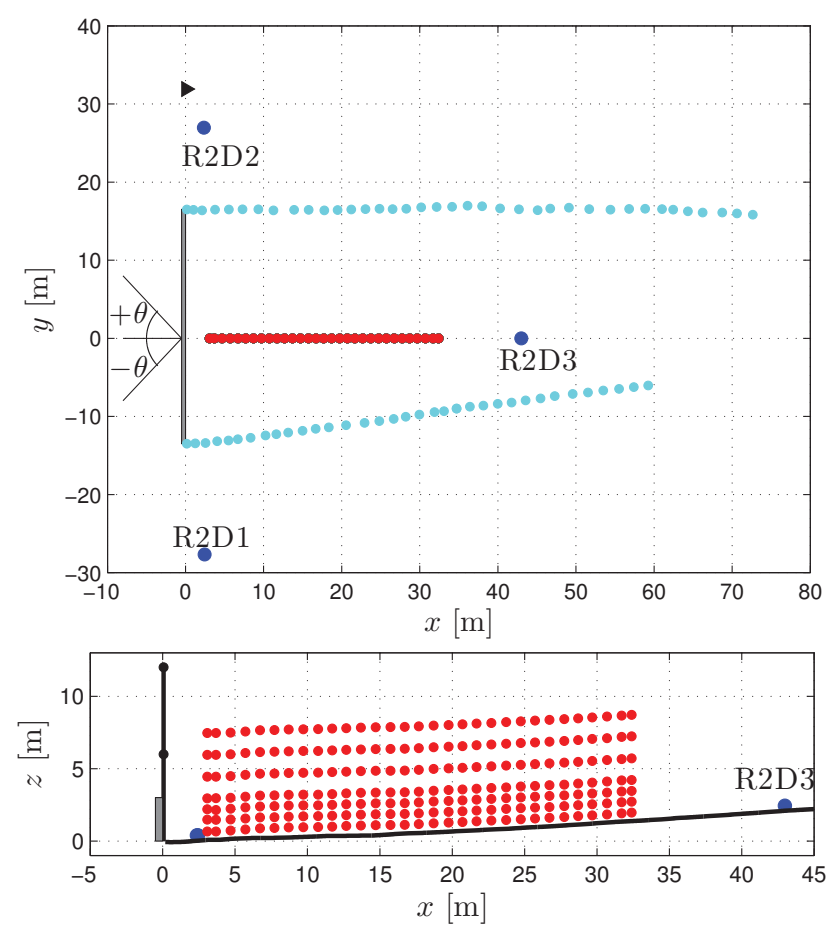

Figure 4. The fence experiment in the reference coordinate system. The positions of the fence (gray rectangle), the lidars (blue circles), the mast (black triangle and black thick line), scanning grid (red circles), and GPS measurements (cyan circles) are also illustrated both at the top (top panel) and side (bottom panel) views. The terrain elevation is also shown in the side view.

Radial speeds are acquired by a CW lidar within a probe volume, whose dimensions are defined by twice the Rayleigh length and the laser beam's cross-section. The Rayleigh length describes the distribution of the laser intensity along the line-of-sight at a given focus distance and increases quadratically with distance (Sonnenschein and Horrigan, 1971). In the scanning pattern used in the experiment, focus distances varied between 10 and $42 \mathrm{~m}$, corresponding to Rayleigh lengths of 0.10 and $1.52 \mathrm{~m}$, respectively. The crosssection of the probe volume is defined by the waist of the laser beam, which is estimated to be in the order of millimetres. 

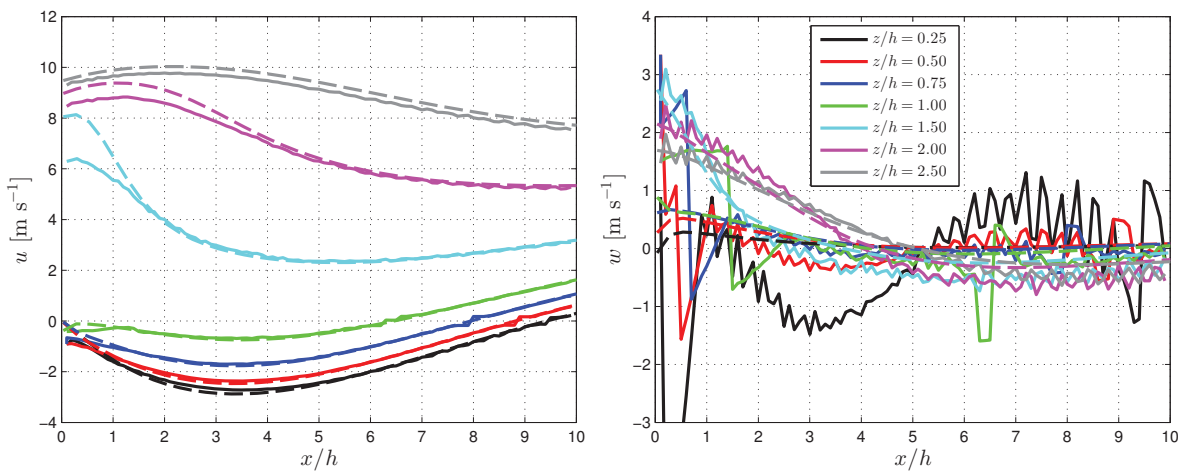

Figure 5. Simulation of the WS measurements (solid lines) and the CFD simulation results (dashed lines) for the $u$ (left panel) and $w$ components (right panel) and several vertical levels.

\subsubsection{WindScanner simulation}

An optimized positioning of the lidars is a compromise between the size of the scanned area, the error in wind speed (which increases with the size of the scanned area), and the wind-speed components (which we are most interested in accurately measuring). As one lidar measures the line-of-sight velocity only, we need to deploy at least one as far downwind as possible, so that under "experimentally ideal" inflow conditions $\left(\theta \approx 0^{\circ}\right)$ this unit measures most of the $u$ component, and as close to the fence to avoid interference of the fence with the probe volume.

A CFD solver of the RANS equations (EllipSys) (Sørensen, 2003) with a standard $k-\varepsilon$ model was used to simulate the flow behind the fence (the solid setup only) and the CFD results were used to "simulate" the flow field observed by the WS including the effect of the probe volume. The CFD simulation was performed using flat terrain with $h / z_{o}=300$. A logarithmic profile in balance with the ground roughness was used as inlet condition for $\theta=0^{\circ}$. To correctly model the high near-fence velocity gradients, the CFD grid had a $0.03 \mathrm{~m}$ wall resolution, which was coarsened with distance to the wall. CFD results were extracted from the same vertical plane as scanned by the WS.

Figure 5 shows both the CFD and the WS's simulated flow assuming that the CFD results "follow" the terrain elevation. The largest differences for the $u$ component occur close to the fence and at $z / h=1.50$ but the relative error is highest for the vertical levels close to the ground. Similarly for $w$, the difference generally increases the closer to the fence and is highest at the two first vertical levels. These are the areas where the CFD simulation results show the highest $w$ gradients and so we expect to have large uncertainty in the $w$ measurements by the WS. A number of positions were tested and the one shown in Fig. 4 and Table 1 was selected because it gave the lowest error for both the $u$ and $w$ components "simulated" by the WS when compared to the CFD results.

\subsubsection{Experimental details}

The scanning pattern on the vertical plane was decided based on the CFD simulation results and the regions where we are interested in measuring the shelter; we want to measure in both the near- and far-wake regions, and below and above $h$ (up to $z / h \approx 2.5$ ). The WS's lidars were therefore set to synchronously scan from a position $1 \mathrm{~m}$ downwind the fence up to a distance of $10 \mathrm{~h}$ and at seven different levels following the terrain elevation. The lidars were continuously acquiring line-of-sight velocity spectra at $\approx 49 \mathrm{~Hz}$. The spectra are gridded in $1 \mathrm{~m}$ cells and spatially averaged in each cell leading to 31 space- and time-averaged spectra per line. The final scanning grid has thus $31 \times 7$ points in the $x-z$ plane. The seven vertical levels are at the heights $[0.21,0.46,0.71,0.96$, $1.46,1.96,2.46] \mathrm{h}$. The 31 positions along the $x$ axis are given at http://www.fence.vindenergi.dtu.dk.

A "full-scan" (a complete measurement of all 217 grid positions), took for most days of the campaign $\approx 21 \mathrm{~s}$. During the second period of the campaign, one lidar had problems with the focus mechanism and to increase the amount of fullscans, we redefine the full-scan on a smaller grid of $29 \times 7$ points, i.e. excluding the grid points furthest and closest to the fence.

After the line-of-sight spectra are averaged in each cell, a series of post-processing steps are performed to first remove noise signals and, subsequently, a median frequency estimator is applied to derive the line-of-sight velocity in each spectrum as in Angelou et al. (2012). As the minimum detectable speed of the WS is $\approx 0.15 \mathrm{~m} \mathrm{~s}^{-1}$, the WS reports a zero lineof-sight velocity for velocities lower than this value. We filter out full-scans where line-of-sight velocities are zero or appear as peaks in the time series (for the latter using the method by Goring and Nikora, 2002) for each lidar and grid position.

The $u, v$, and $w$ components are estimated at each grid position from the scan geometry combined with the line-ofsight velocities. A preliminary analysis of the estimations of $w$ at the first two vertical levels shows unrealistic values 

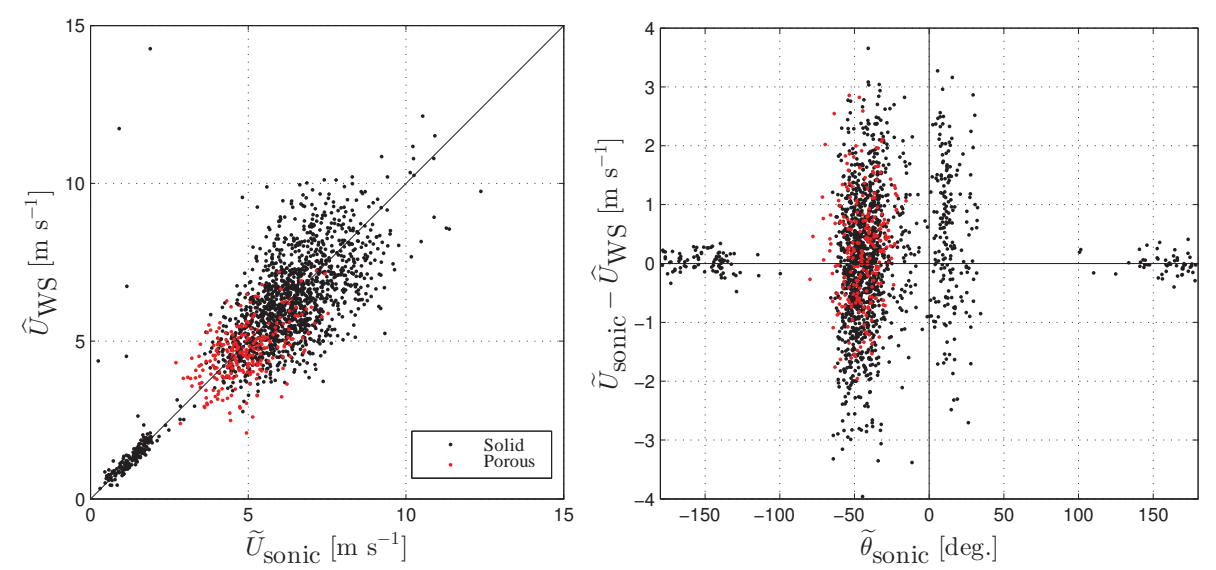

Figure 6. Left panel: scatter plot of wind-speed measurements from the $6 \mathrm{~m}$ sonic anemometer and the WS for the grid point closest to the fence and at a height of $\approx 6 \mathrm{~m}$. Right panel: the difference between these two measurements as function of the relative wind direction observed by the $6 \mathrm{~m}$ sonic anemometer.

because the line-of-sight of the lidars is almost perpendicular to $w$. Therefore, for all the positions in these two levels, we use the line-of-sight velocities of R2D1 and R2D3 only, so at these two levels we can only estimate $u$ and $v$ assuming a zero $w$ component.

The WS was mostly operated when the sonic anemometer measurements indicated westerlies and during periods without rain. The WS measurements are thus concentrated on few days as indicated in Table 2, which shows the amount of fullscans per day.

\section{Data analysis}

\subsection{Sonic anemometer-lidar intercomparison}

Besides the 10 min mean and turbulence sonic anemometer statistics, we derive another set of statistics based on the time period that the WS takes to complete each full-scan (denoted by a $\sim$ symbol). Thus, we also know both the mean wind speed and direction, and their variability, within this shorter period.

The grid point closest to the sonic anemometer at $6 \mathrm{~m}$ is at a height of $\approx 6 \mathrm{~m}$. We compare the WS measurements at this grid position with those from the $6 \mathrm{~m}$ sonic anemometer. This is not a fair comparison because the measurements from the WS at each grid position are nearly "instantaneous", i.e. it takes less than $0.1 \mathrm{~s}$ to scan each grid point (we use $\mathrm{a}^{-}$symbol to refer to them), whereas we use the full-scan period for the sonic anemometer measurements. However, the comparison shows us the conditions in which the flow at both positions is similar. Figure 6 shows a scatter plot of such measurements for both periods of the campaign.

Figure 6 (left panel) illustrates the good agreement between the $6 \mathrm{~m}$ sonic anemometer and the WS for the horizontal wind-speed magnitude; the scatter is low and high for low and high wind speeds, respectively. Figure 6 (right panel)
Table 2. Number of full-scans per day by the WS and the fence porosity.

\begin{tabular}{lrl}
\hline Date & No. of full-scans & Porosity \\
\hline 10 March & 637 & solid \\
11 March & 712 & solid \\
20 March & 11 & solid \\
26 March & 84 & solid \\
27 March & 81 & solid \\
1 April & 27 & solid \\
30 September & 11 & porous \\
1 October & 107 & porous \\
2 October & 125 & porous \\
\hline
\end{tabular}

shows that the degree of scatter is a function of the relative wind direction; when the WS measures downwind the fence $\left(\left|\widetilde{\theta}_{\text {sonic }}\right| \leq 90^{\circ}\right)$, the scatter is much higher than that for upwind conditions. Although the grid point used is $\approx 1 h$ above and $\approx 1 h$ downwind the fence, there seems to be a strong effect of the fence on the flow at this position, whereas the effect is nearly negligible for $\left|\widetilde{\theta}_{\text {sonic }}\right| \geq 90^{\circ}$. A similar analysis for downwind conditions using the grid point furthest away from the fence $(\approx 32 \mathrm{~m})$ and at the same vertical level shows a reduction of the scatter (not shown) as the shelter is low there (see Sect. 5). Figure 6 (right panel) also shows that most of the measurements are concentrated at $\widetilde{\theta}_{\text {sonic }} \approx-50^{\circ}$ and that few winds are normal to the fence.

\subsection{Inflow conditions}

The flow at the mast position (assumed to be undisturbed by the fence for $-75 \leq \theta \leq 75^{\circ}$ ) determines the inflow conditions required to estimate the wind-speed ratio due to shelter. Therefore, we need to estimate the surface conditions as a function of relative wind directions at the site. 
Table 3. Roughness length $z_{o}$ as function of the relative wind direction $\theta$ based on either the 6 or the 12 m sonic anemometer for both March and September 2015. The amount of $10 \mathrm{~min}$ samples is also shown.

\begin{tabular}{lccrcrr}
\hline$\theta \pm 5^{\circ}$ & $6 \mathrm{~m} z_{o}[\mathrm{~m}]$ & $\begin{array}{c}\text { March } \\
12 \mathrm{~m} z_{o}[\mathrm{~m}]\end{array}$ & $\begin{array}{r}\text { No. of 10 min } \\
\text { samples }\end{array}$ & $\begin{array}{r}\text { September } \\
6 \mathrm{~m} z_{o}[\mathrm{~m}]\end{array}$ & $\begin{array}{r}\text { No. of 10 min } \\
\text { samples }\end{array}$ \\
\hline-90 & 0.0673 & 0.0785 & 176 & 0.0549 & 0.1204 & 184 \\
-80 & 0.0435 & 0.0542 & 174 & 0.0280 & 0.0574 & 193 \\
-70 & 0.0231 & 0.0173 & 174 & 0.0095 & 0.0143 & 155 \\
-60 & 0.0095 & 0.0070 & 116 & 0.0072 & 0.0089 & 95 \\
-50 & 0.0069 & 0.0095 & 114 & 0.0052 & 0.0068 & 79 \\
-40 & 0.0049 & 0.0075 & 148 & 0.0028 & 0.0048 & 120 \\
-30 & 0.0031 & 0.0051 & 183 & 0.0012 & 0.0024 & 108 \\
-20 & 0.0031 & 0.0036 & 61 & 0.0009 & 0.0014 & 70 \\
-10 & 0.0021 & 0.0033 & 40 & 0.0004 & 0.0005 & 74 \\
0 & 0.0014 & 0.0010 & 33 & 0.0009 & 0.0018 & 118 \\
10 & 0.0013 & 0.0026 & 61 & 0.0018 & 0.0064 & 140 \\
20 & 0.0014 & 0.0051 & 15 & 0.0030 & 0.0046 & 106 \\
30 & 0.0015 & 0.0043 & 43 & 0.0060 & 0.0084 & 93 \\
40 & 0.0020 & 0.0038 & 16 & 0.0121 & 0.0077 & 47 \\
50 & 0.0113 & 0.0447 & 10 & 0.0407 & 0.0536 & 15 \\
60 & 0.0280 & 0.0859 & 12 & 0.0975 & 0.2840 & 11 \\
70 & 0.0204 & 0.0778 & 34 & 0.0172 & 0.0641 & 1 \\
80 & 0.0149 & 0.0970 & 55 & 0.0151 & 0.1155 & 7 \\
90 & 0.0330 & 0.2586 & 73 & 0.0289 & 0.3818 & \\
\hline
\end{tabular}

Table 4. Case studies for a number of $\theta$ intervals. Refer to the text for details.

\begin{tabular}{llrrlrr}
\hline Case & Porosity & $\theta$ [deg.] & $\left\langle z_{o}\right\rangle[\mathrm{m}]$ & $u_{* \text { est }}\left[\mathrm{m} \mathrm{s}^{-1}\right]$ & $\langle z / L\rangle$ & $\begin{array}{r}\text { No. of } \\
\text { full-scans }\end{array}$ \\
\hline I & solid & $0 \pm 15$ & 0.0016 & 0.36 & 0.021 & 159 \\
II & solid & $0 \pm 30$ & 0.0019 & 0.36 & 0.015 & 304 \\
III & solid & $-30 \pm 15$ & 0.0037 & 0.34 & 0.023 & 604 \\
IV & solid & $-60 \pm 15$ & 0.0131 & 0.39 & 0.045 & 583 \\
V & solid & $30 \pm 15$ & 0.0016 & 0.35 & 0.007 & 62 \\
VI & solid & $0 \pm 30$ & 0.0019 & $0.28,0.27$ & 0.044 & 92 \\
VII & porous & $-30 \pm 15$ & 0.0016 & 0.25 & -0.068 & 128 \\
\hline
\end{tabular}

Assuming homogenous inflow over flat terrain, we estimate $z_{o}$ from Eq. (1) using 10 min mean and turbulence sonic anemometer statistics. Thus, we have two $z_{o}$ values derived using either sonic anemometer for each $10 \mathrm{~min}$ period. To compute $\psi_{m}$, we use the forms in Peña (2009). Table 3 shows the median of such $z_{o}$ estimations based on the 6 and $12 \mathrm{~m}$ sonic anemometers for March and September 2015 and for $10^{\circ} \theta$ intervals (we use the $10 \mathrm{~min}$ mean sonic anemometer wind direction $\bar{\theta}_{\text {sonic }}$ to classify the $10 \mathrm{~min}$ sonic anemometer statistics into relative direction intervals). As shown, for both periods, $z_{o}$ increases with increasing $|\theta|$, as expected, due to the topography upstream the fence (see Fig. 2). Further, the difference in $z_{o}$ values between both periods is relatively small indicating that, particularly at $\theta \approx 0^{\circ}, z_{o}$ is greatly influenced by the fjord's surface conditions.

We need to find out if it is sufficient to describe the inflow using Eq. (1) with the $z_{o}$ values in Table 3 given that the terrain is not flat, the upstream conditions not homogeneous, and the atmospheric conditions generally not neutral. For this, we define "case" studies based on $\theta$ intervals and we select the data, which are included in each case, using the full-scan mean direction from the $6 \mathrm{~m}$ sonic anemometer, i.e. $\widetilde{\theta}_{\text {sonic }}$ (see Table 4).

The case studies are selected so that each has a significant number of full-scans and that we can study the influence on the shelter of a wider $\theta$ interval (cases I and II), $\theta$ itself (cases I and III-V), atmospheric stability (cases II and VI), and porosity (case VII). Table 4 provides an estimation of different parameters that are used to reproduce the inflow conditions for each case, which are also illustrated in Fig. 7 (left panel). For each case, we

1. average the $z_{o}$ values in Table 3 within the $\theta$ interval in Table 4 (we denote this type of ensemble average with the \langle\rangle symbol); 

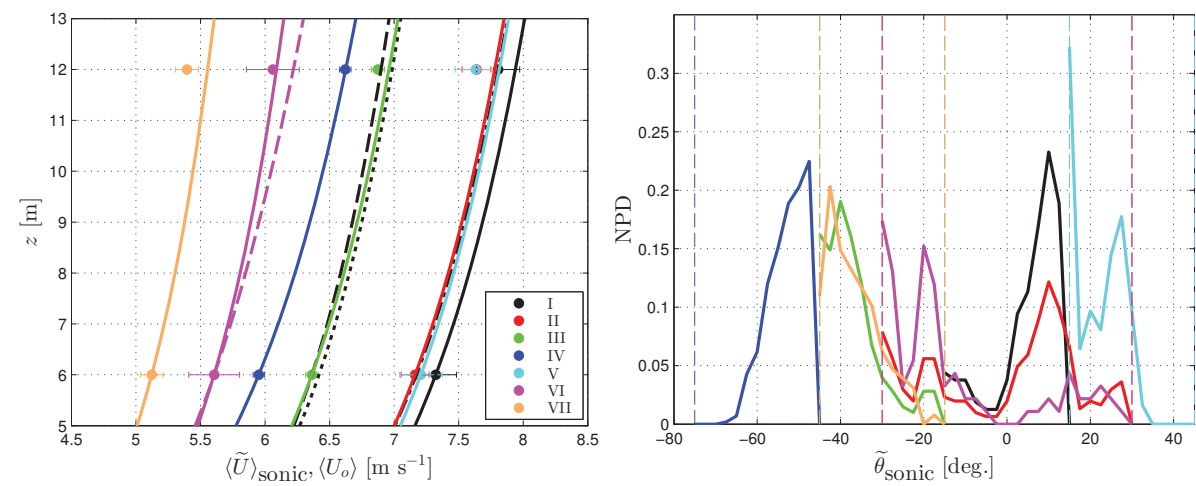

Figure 7. Left panel: inflow conditions for the case studies. The circle markers indicate the ensemble-averaged sonic anemometer measurements $\langle\widetilde{U}\rangle_{\text {sonic }}$ ( \pm the standard error in the error bar) and the lines the estimations of the mean inflow conditions $\left\langle U_{o}(z)\right\rangle$ (see text for details). Right panel: normalized distribution (NPD) of the relative wind direction from the $6 \mathrm{~m}$ sonic anemometer $\widetilde{\theta}_{\text {sonic }}$ for the case studies.

2. estimate a "new" friction velocity $u_{* \text { est }}$ with Eq. (1) assuming $\psi_{m}(z / L)=0$ and using the sonic anemometer wind-speed measurement at $6 \mathrm{~m}$, ensemble-averaged from the sonic anemometer mean wind speeds within the full-scan period,

$$
u_{* \mathrm{est}}=\frac{\kappa\langle\widetilde{U}\rangle_{\text {sonic }}}{\ln \left(6 \mathrm{~m} /\left\langle z_{o}\right\rangle\right)},
$$

3. estimate the "mean" inflow wind profile $\left\langle U_{o}(z)\right\rangle^{2}$ using Eq. (1) assuming $\psi_{m}(z / L)=0$ (solid colour lines in Fig. 7, left panel) as

$$
\left\langle U_{o}(z)\right\rangle=\frac{u_{* \mathrm{est}}}{\kappa} \ln \left(\frac{z}{\left\langle z_{o}\right\rangle}\right) .
$$

As shown, the estimations of the inflow profiles are in good agreement with the sonic anemometer measurements (an absolute error of $0.18 \mathrm{~m} \mathrm{~s}^{-1}$ is computed at $12 \mathrm{~m}$ for case $\mathrm{V}$ as the largest of all cases). We therefore assume that, although present, the topographic effects at the mast position within the heights 6-12 $\mathrm{m}$ can be neglected for these $\theta$ ranges. The inflow is thus well described by the logarithmic profile.

In addition, Fig. 7 (left panel) shows three more profiles for case I. The black dashed line shows the mean inflow conditions but using the ensemble-average $u_{*}$ of $u_{*}$ values estimated from the $6 \mathrm{~m}$ sonic anemometer with Eq. (2) within the full-scan period, i.e. $\left\langle\tilde{u_{*}}\right\rangle$. In this case, there is a systematic underestimation of the inflow wind speed because $\left\langle\tilde{u_{*}}\right\rangle$ is about $13 \%$ lower than $u_{* \text { est }}$ (the latter is given in Table 4). The results in the black dash-dotted line are obtained similarly to those in the solid lines but using the $12 \mathrm{~m}$ sonic anemometer and the $\left\langle z_{o}\right\rangle$ derived from the observations

\footnotetext{
${ }^{2}$ Although this is not an ensemble average per definition, we use the \langle\rangle symbol because it results from the ensemble-averaged roughness length $\left\langle z_{o}\right\rangle$.
}

at that height. Therefore, the estimated inflow wind speed at $12 \mathrm{~m}$ is equal to the ensemble-average sonic anemometer wind speed at the same height. The results in the black dotted line are found with the same methodology as that used for the results in the dashed line but with the $12 \mathrm{~m}$ sonic anemometer. From these three results, we confirm the following: first, that turbulent fluxes estimated in the short period of the fullscan are not adequate for deriving the inflow conditions (see the work of Lenschow et al., 1994) and, second, that similar results are obtained when using $z_{o}$ estimations based on either the 6 or $12 \mathrm{~m}$ sonic anemometer. This also gives us an idea of the small effect that the internal boundary layer (developed at the coastline) has on the inflow profile at the mast position and within the heights between the sonic anemometers.

For case VI, a second mean inflow profile (magenta dashed line) is shown in Fig. 7 (left panel). Case VI is similar to case II but we narrow the analysis to stable conditions $z / L \geq 0.01$ from the "concurrent" $10 \mathrm{~min}$ derived turbulence sonic anemometer estimates at $6 \mathrm{~m}$. $u_{* \text { est }}$ can be computed as in Eq. (10) and, in addition, the correction due to atmospheric stability can be included (the result is the second value for the $u_{* \text { est }}$ column in Table 4). Thus, the magenta dashed line shows the mean inflow profile using Eq. (11) with this new $u_{* \text { est }}$ value, which overestimates the mean wind speed at $12 \mathrm{~m}$ by $0.16 \mathrm{~m} \mathrm{~s}^{-1}$ only.

For each case in Table 4, we include the average dimensionless stability $\langle z / L\rangle$ value, which is found by averaging (in an ensemble sense) the 10 min turbulence fluxes from the $6 \mathrm{~m}$ sonic anemometer that are "concurrent" with the time of the full-scans. As shown, the atmosphere for the "solid fence" cases is on average stable, except for case V, which corresponds to the most northern winds, and for the "porous fence" case the atmosphere is unstable. Interestingly, although we do not narrow the filtering criteria to stable con-

\footnotetext{
${ }^{3}$ Quotation marks because a full-scan takes less than $10 \mathrm{~min}$.
} 

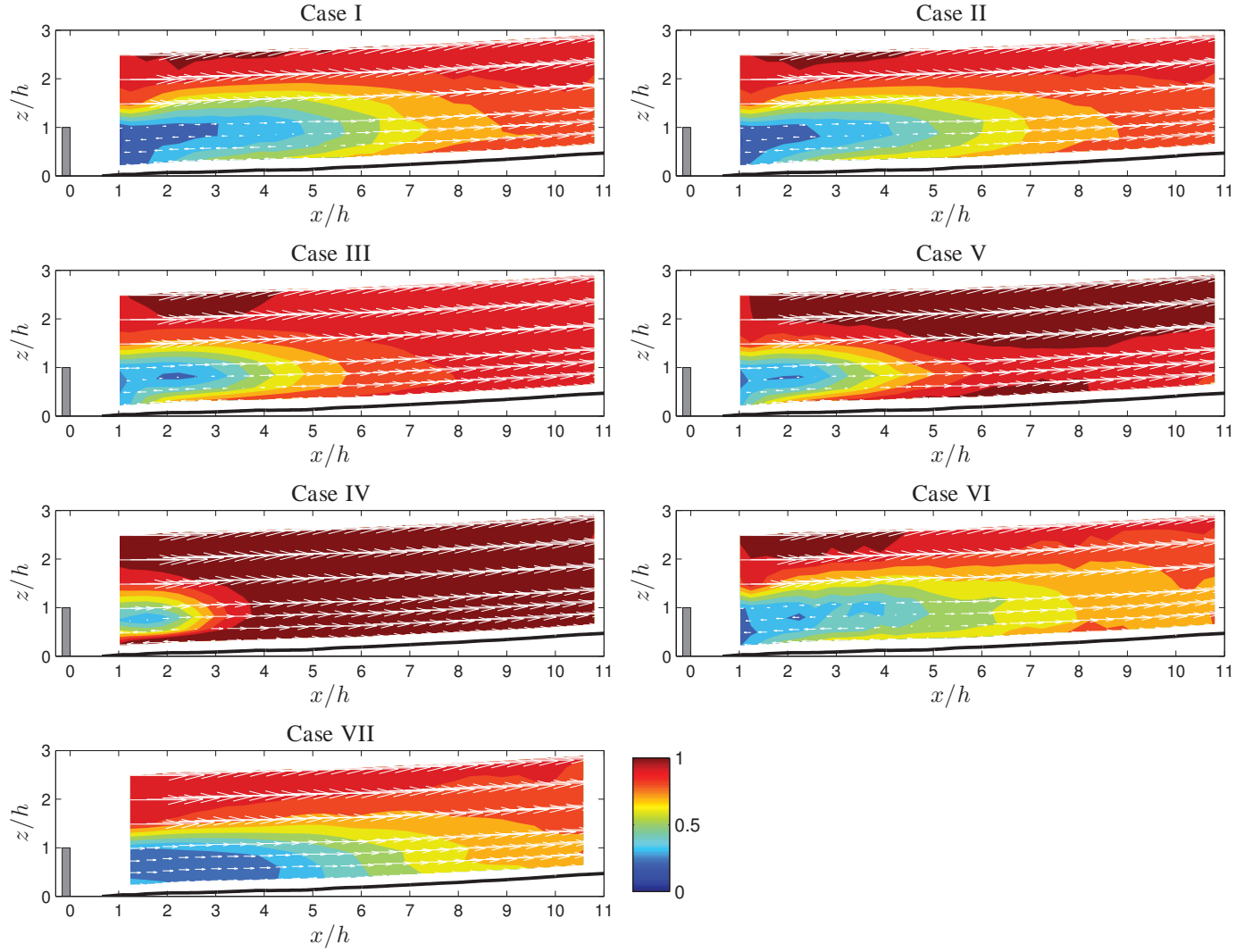

Figure 8. Averaged wind-speed ratio $\langle\widetilde{U}(x, z) \mathrm{WS}\rangle /\left\langle U_{o}(z)\right\rangle$ (colourbar) behind the fence for a number of cases. Vectors indicate the magnitude and sign of the ensemble-averaged $u$ component.

ditions for case IV, $\langle z / L\rangle$ is higher for this case than for case VI.

Figure 7 (right panel) shows that the distribution of $\tilde{\theta}_{\text {sonic }}$ values for each case is not uniform and that the centre of the interval, in most cases, differs from the mean of the relative directions within the interval; thus these distributions should be taken into account when evaluating models. We provide the values of such distributions at http://www. fence.vindenergi.dtu.dk.

\section{Results}

\subsection{Wind-speed ratio}

We classify the data from the WS's full-scans into the cases in Table 4 using the $\widetilde{\theta}_{\text {sonic }}$ values. The horizontal velocities from the WS are then ensemble-averaged within each case, $\left\langle\widetilde{U}(z)_{\mathrm{Ws}}\right\rangle$, and the wind-speed ratio is estimated by normalizing these averages by the case-correspondent "mean" inflow profile (described in Sect. 4.2).

The plots in Fig. 8 illustrate the wind-speed ratio for each case. Although $\widetilde{\theta}_{\text {sonic }}$ is not uniformly distributed within the chosen relative direction intervals, the effect of the fence on the flow for varying $\theta$ values is well observed, particularly from the results between cases I, III and IV (three left frames from the top). Case I, as expected, seems to have the deepest shelter effect of these three cases, which diminishes when increasing $|\theta|$ and, for case IV, the effect of the fence is only noticed for $x / h \lesssim 3$. For case II, which is defined similar to case I but for a broader $\theta$ interval, the effect of the fence on the flow is smoother and seems slightly deeper than that for case I but the differences are not large. This is most probably due to the concentration of full-scans at $\widetilde{\theta}_{\text {sonic }} \approx 10^{\circ}$ in both cases. Case VI, the "stable" case II, also shows a similar behaviour but with slightly deeper shelter effects than case II. Case V, similarly defined as case III but with $\theta$ centred at $30^{\circ}$, shows reductions up to $50 \%$ for $x / h \lesssim 4$ as case III also does. Case VII, which is comparable to case III but for a different porosity, does not show wind-speed ratios close to zero but the shelter seems to extend further away from the fence.

For cases I-III and VI we notice a small region where the wind-speed ratio is larger than one, located at $x / h \approx 2.5$ and $z / h \approx 2.5$. High wind-speed ratios within the range $1 \leq x / h \leq 4$ are also observed for some of the other cases in Figs. 9-11. These figures illustrate the behaviour of the windspeed ratio but separated into the $u$ and $v$ components, with distances downwind the fence, for the seven different levels, and for the seven cases. For $z / h=2.46$ (Fig. 11), a $u$ "bump" 

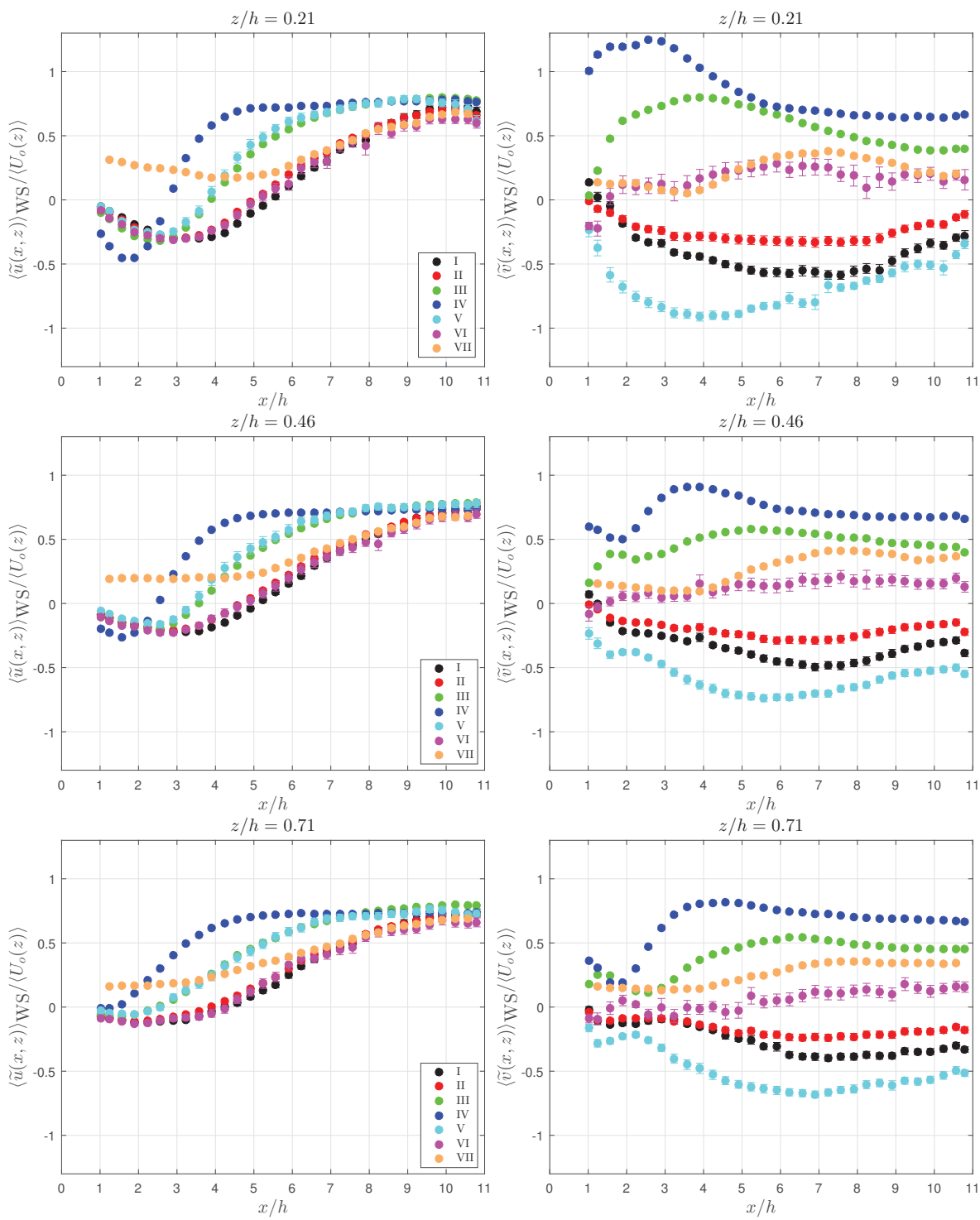

Figure 9. Averaged wind-speed ratio (separated into the $u$ and $v$ components) on the first three verticals level behind the fence for a number of cases. \pm the standard error is shown in the error bars.

is clearly visible for all cases where the fence is solid (it is also present on the data of the porous setup). In Fig. 12, we show this high wind-speed ratio from the CFD simulation (used to estimate the WS's error in Sect. 3.3). The CFD simulation was performed over flat terrain without roughness changes and so it is the fence itself that causes the increased vertical velocity shear. Further, the results in Figs. 9-11 for case IV, in which the inflow is the most oblique, show that both $u$ and $v$ components do not largely vary for $x / h \geq 7$. This shows us that the effect of the topography on the flow is small at all scan positions on the vertical plane relative to that at the mast position (the wind-speed ratio based on $U$ is $\approx 1$ ).

In Fig. 8, the sign and magnitude of the ensemble-averaged WS's $u$ component is also illustrated. A region of reverse flow is visible for all cases when the fence is solid; this is also seen on the left frames of Figs. 9-11. This region is also shown in Fig. 12 but for the CFD results it extends much further downwind because the simulation is performed for $\theta=0^{\circ}$ only.

The results in Figs. 9-11 confirm those in Fig. 8; for the solid setup, case VI generally shows the highest shelter in the far wake when looking at the $u$ component, systematically followed by cases II, I, III, V, and IV, as expected, due to the relative inflow directions, a ranking supported by the proximity of the $v$ component to zero for each of the cases. Interestingly, the behaviour of the $u$ component for case VII follows that of cases I, II and VI in the far wake, and does not strongly vary below $h$ in the near-wake region; case VII is the only case where the $u$ component does not change sign, 

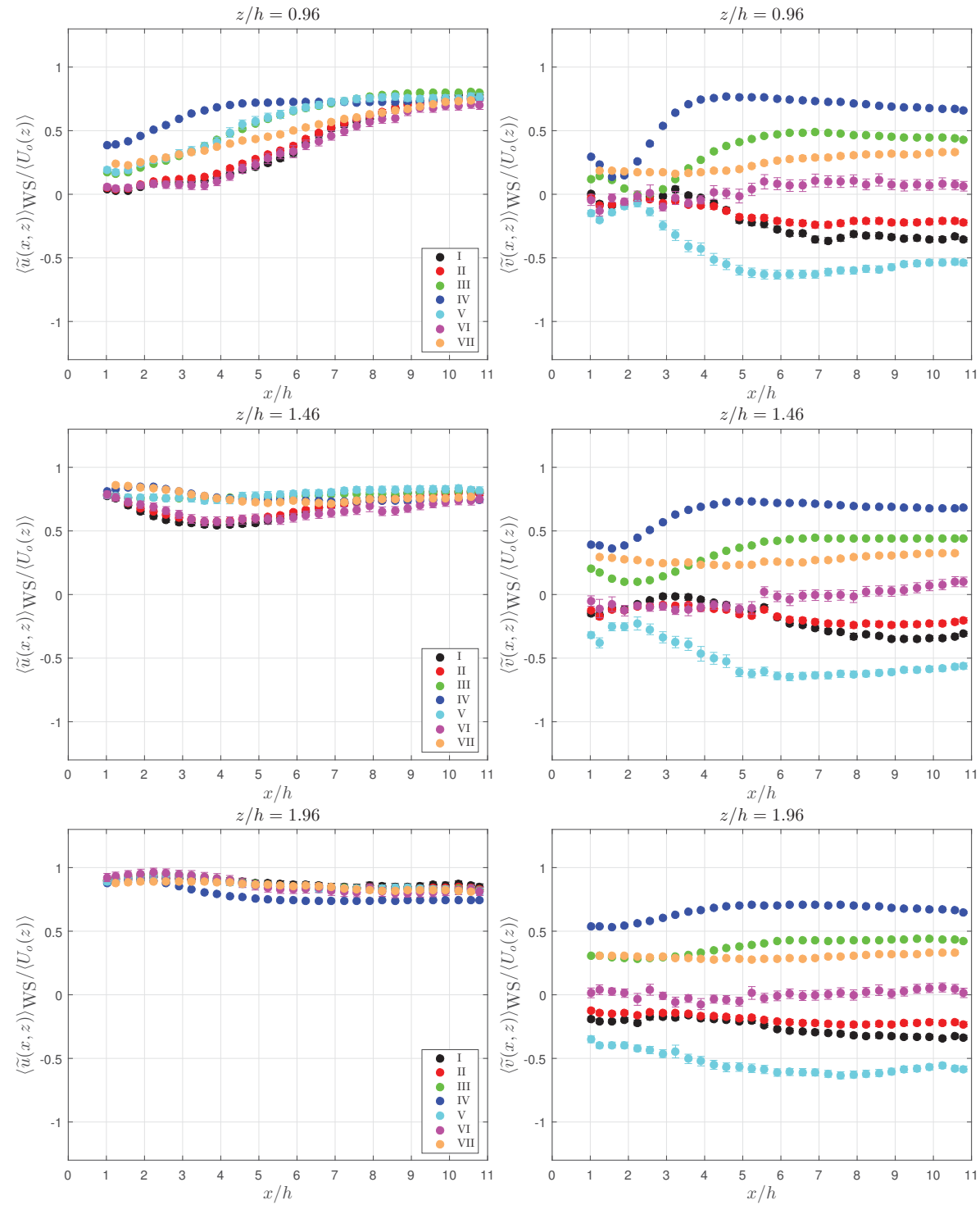

Figure 10. Same as Fig. 9 but for $z / h=0.96,1.46$, and 1.96.
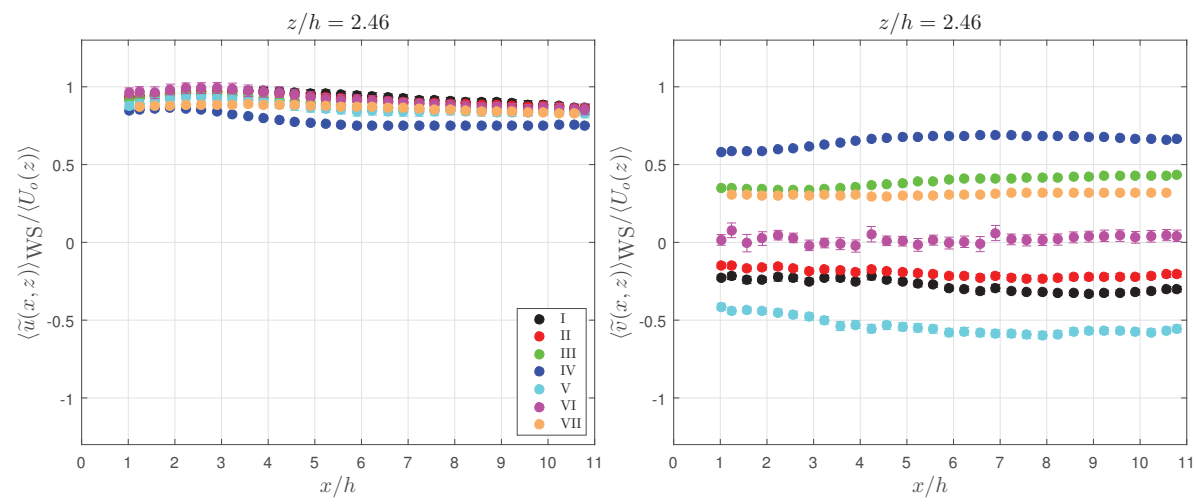

Figure 11. Same as Fig. 9 but for $z / h=2.46$. 


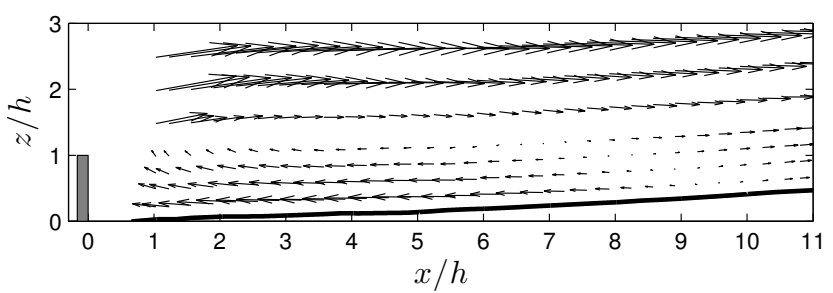

Figure 12. Velocity vector downwind the fence based on the CFD simulation for $\theta=0^{\circ}$.

i.e. no reverse flow. For all the other cases, reverse flow can be distinguished and vanishes only at $z / h \geq 0.96$. The behaviour of the $u$ component with distance from the fence is similar for cases I, II and VI, although the differences in the cases' obliquities are evident when looking at the $v$ component. It is interesting to note that for the porous case corresponding to oblique flow, at the two first vertical levels and for $x / h \lesssim 4$, the horizontal wind vector does not rotate much compared to the inflow condition; the $v$ component seems to be less effectively reduced compared to the solid cases. For the latter cases the horizontal wind vector strongly rotates downwind of the fence.

Cases III and V show a similar behaviour for the $u$ component; case V systematically showing less shelter, which is explained by the slightly higher proximity to zero of the $v$ component in case III. In both cases, the $v$ component behaves remarkably similar but with opposite sign at all vertical levels. The average wind-speed ratio for both $u$ and $v$ components as function of distance from the fence and for each level and case is presented at http://www.fence.vindenergi.dtu.dk.

\subsection{Self-preserving velocity profiles}

Using observations from three of the cases in which the $\theta$ interval is centre at $0^{\circ}$, we compute the self-preserving forms (Sect. 2) and illustrate them (Fig. 13). We

1. estimate a "mean" shear exponent $\langle n\rangle$ using the caseconcurrent ensemble-average sonic anemometer measurements and the power law,

$$
\langle n\rangle=\frac{\ln \left[\langle\tilde{U}\rangle_{\text {sonic }}(z=6 \mathrm{~m}) /\langle\tilde{U}\rangle_{\text {sonic }}(z=12 \mathrm{~m})\right]}{\ln (6 \mathrm{~m} / 12 \mathrm{~m})},
$$

2. compute a "mean" $K$ using the average roughness (Table 4$),\langle K\rangle=2 \kappa^{2} / \ln \left(h /\left\langle z_{o}\right\rangle\right)$,

3. use the estimations of the mean inflow (Eq. 11) at the vertical levels and at $z=h$ to compute the average selfsimilar profiles,

$$
\frac{\langle\Delta U(x, z)\rangle}{\left\langle U_{o}(h)\right\rangle}\left(\frac{x}{h}\right)=\frac{\left\langle U_{o}(z)\right\rangle-\langle\widetilde{U}(x, z)\rangle_{\mathrm{WS}}}{\left\langle U_{o}(h)\right\rangle}\left(\frac{x}{h}\right),
$$

4. estimate a "mean" $\eta$ value, $\langle\eta\rangle$, based on Eq. (7) using $\langle K\rangle$ and $\langle n\rangle$.
Figure 13 shows the self-preserving profiles for a number of downwind distances; near-wake profiles $(x / h<5.6)$ in grey markers and far-wake profiles $(x / h>6.24)$ in nongrey circles. Equation (8) with $A=9.75$ is also shown. Further, we fit Eq. (9) to the far-wake profiles, where $C_{h}$ is estimated in a least-squares sense.

The profiles in the near wake do not generally converge onto each other, whereas those in the far wake do, particularly for cases II and VI with the broad direction interval. Equation (9) with the adjusted $C_{h}$ agrees better with the profiles compared with Perera's expression in Eq. (8), particularly where the term $\left[\langle\Delta U(x, z)\rangle /\left\langle U_{o}(h)\right\rangle\right](x / h)$ peaks (vertical levels below $h)$, due to the low $C_{h}$. For these cases, Perera's estimations result in a general overestimation of the wind-speed ratio below $h$.

The adjusted $C_{h}$ value in Eq. (9) changes considerably for these cases. For the narrow direction interval (case I), it is nearly half the value recommended by Taylor and Salmon for 2-D fences and increases the broader the interval. The increase of $C_{h}$ in case II compared to case I can be explained by the $\theta$ distribution in Fig. 7 (right panel); the ensembleaverage relative direction in case I is $6.27^{\circ}$ and in case II is $0.39^{\circ}$, which partly explains the larger effect of the fence on the flow for case II (see also the differences in the $v$ component in Figs. 9-11). The effect on the flow is larger in case VI; the stable conditions might be responsible for the increase in $C_{h}$ and the deeper wake but this is also the case where the $v$ component is closest to zero.

\section{Conclusions and discussion}

Full-scale flow measurements on a vertical plane behind a fence are presented. The measurements were conducted by the WS and agree well with sonic anemometer measurements from a nearby mast when the wind is not largely disturbed by the fence. Simulation of the WS measurements reveals that the WS tends to underestimate the magnitude of the $u$ component at $x / h \lesssim 4$ and $z / h>1$. This is mostly due to the combination of the high vertical velocity gradient and the large probe volume of the lidar furthest downwind from the fence.

The wind-speed ratio (defined by either $u, v$, or $U$ with respect to the inflow) depends on the inflow conditions. We assume the topographic effects at each of the positions on the vertical plane to be similar to those at the mast position at the same height, as the wind-speed ratio (based on $U$ ) approaches one for the case where the inflow is the most oblique (case IV) at $x / h \gtrsim 6$ and for all vertical levels. Between the sonic anemometers $(6-12 \mathrm{~m})$, the inflow conditions are well described by the logarithmic profile using direction-dependent roughness values estimated from the 10 min sonic anemometer observations. Orographic effects can thus be negligible at the mast (between sonic anemometers) but the effect of the sea-to-land roughness change up- 

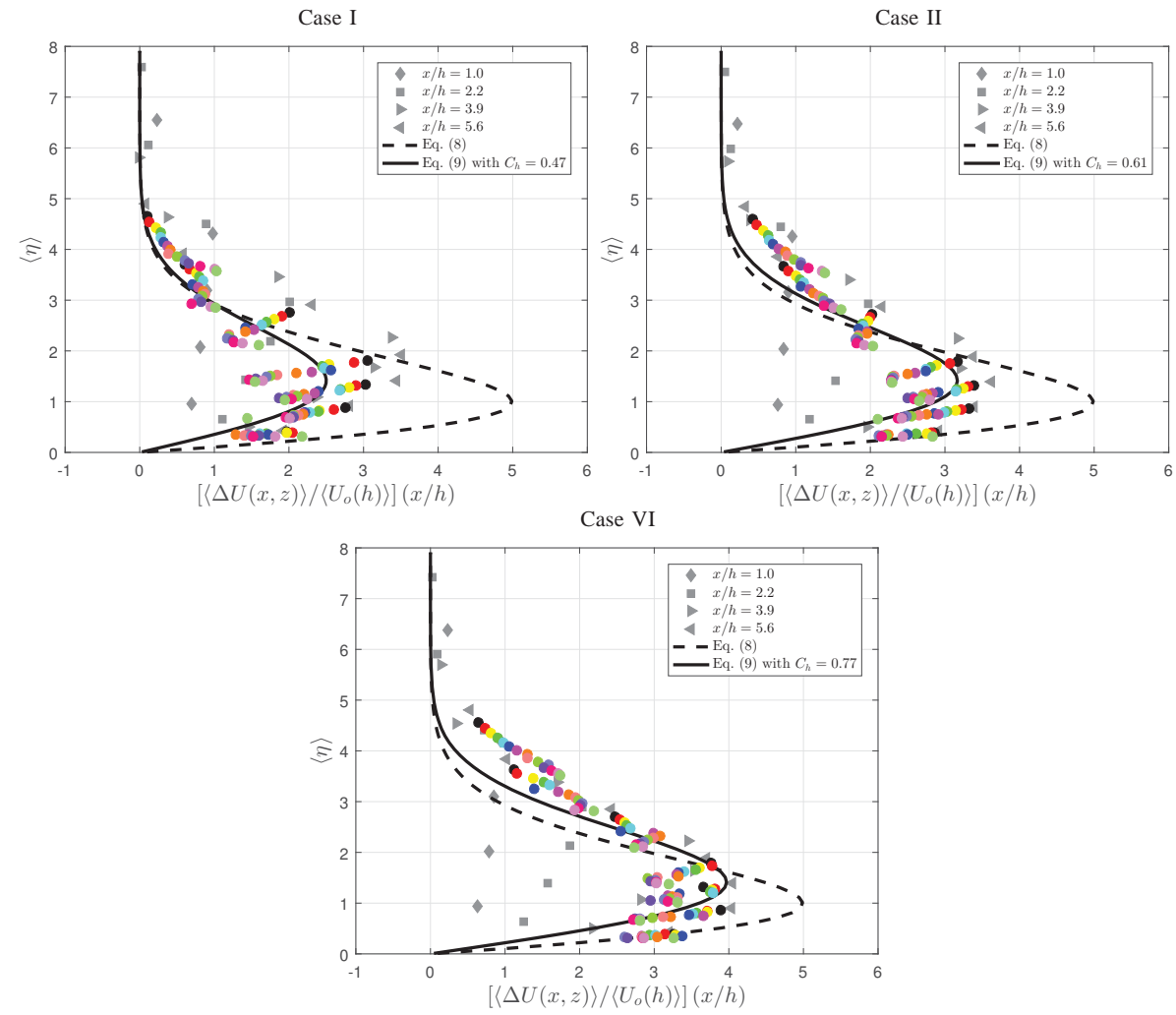

Figure 13. Self-preserving profiles for three cases and a number of downwind distances (details in the text). Results from Eqs. (8) and (9) are also shown.

wind the fence is perhaps important. Inflow conditions derived from the sonic anemometers are related mostly to the flow characteristics upwind the closest sea-to-land roughness change; the wind profile is in equilibrium with the new surface the first $\approx 1 \mathrm{~m}$ only. When evaluating flow models, topographic effects can be added. We provide the data to derive the inflow conditions that we use to compute the wind-speed ratios and so other inflow conditions can be used if preferred. All data are available at http://www.fence.vindenergi.dtu.dk.

The wind-speed ratio follows the expected behaviour; for increasing relative directions and in the far wake, the flow is less disturbed by the fence and within the near-wake region, the porous fence has a lower effect on the flow than the solid fence. For model evaluation, the relative direction distribution needs to be taken into account, as its effects are obvious. We observe a deeper effect of the fence on the flow in the stable compared to the near-neutral case with the same relative-direction interval; model comparison is encouraged to distinguish if this is a result of stability or of the relativedirection distribution. For all cases, the fence decreases the $u$ component for $z / h \leq 1.5$, and for some cases and levels the fence speeds up the flow.

Direct comparisons with previous experiments are difficult to perform due to differences in, e.g. the nature of the obstacle, the inflow conditions, the accuracy of the mea- surements, and the shelter regions analysed. The shelter observations studied by Wilson (2004) for $z / h=0.5$ and at $x / h=2,4,6$ and 10 for $\theta=0^{\circ}$ (with $\varphi=0.45$ ) show windspeed ratios (based on $U$ ) of $\approx 0.48,0.44,0.48$, and 0.64 , respectively, whereas for our porous setup $(\varphi=0.375)$ these are $0.25,0.27,0.45$, and 0.78 , i.e. half the values in the near wake and similar ones in the far wake. It is important to mention that the values in Wilson (2004) are the average of cup-anemometer wind speeds within one $15 \mathrm{~min}$ period only. A more porous windbreak $(\varphi=0.50)$ is studied in Seginer (1975) and the wind-speed ratios for $\theta=0^{\circ}$ at $z / h=0.25$ and $x / h \approx 2.5,5$, and 7.5 are $\approx 0.4,0.3$, and 0.35 (for adiabatic conditions), whereas ours (at $z / h=0.21$ ) are 0.28 , 0.31 , and 0.60 . They only agree at $x / h=5$ and the difference at $x / h=2.5$ is expected due to porosity; Seginer (1975) finds much more reduced shelter with instability in the far wake (our porous case corresponds to an unstable atmosphere). Also important to note is that in both studies the accuracy of the measurements is a major concern. Cup anemometers do not respond well for increasing flow angles and both cup and sonic anemometers can be subjected to large flow distortion when mounted on masts. We recommend that for future shelter experiments, lidar measurements (which also have inaccuracies) are supplemented by those from cups and sonic anemometers. In our particular case, turbulence is difficult 
to address with the WS measurements alone as we do not acquire radial velocities at the same grid point for a longenough period.

The velocity-deficit profiles within the far-wake region $(x / h>6.24)$ converge onto each other, although proper comparison with Counihan et al. (1974) theory can only be made with a much wider fence. The Counihan et al. (1974) solution agrees better with the self-preserving profiles than Perera's expression, which overestimates the effect of the fence on the flow at $z / h \leq 1$. This is mainly due to the low wake-momentum coefficient when compared to that used for 2-D obstacles. Counihan et al. (1974) and Perera (1981) works are mostly based on wind-tunnel studies for flow nearly perpendicular to the obstacle. Model evaluation with our measurements could provide insights about accounting for 3-D effects on analytical solutions and the wakemomentum coefficient dependency on relative directions.

Acknowledgements. Funding from EUDP, Denmark to both the IEA Task 27 "small turbines in high turbulence sites" and the "Online WAsP" project (www.mywindturbine.com) are acknowledged.

Edited by: J. Lundquist

Reviewed by: P. A. Taylor and E. S. Takle

\section{References}

Angelou, N., Abari, F. F., Mann, J., Mikkelsen, T. K., and Sjöholm, M.: Challenges in noise removal from Doppler spectra acquired by a continous-wave lidar, in: Proc. 26th International Laser Rader Conf: Session 5P: Atmosp. winds - turbulence, Porto Heli, 2012.

Castro, I. P. and Robins, A. G.: The flow around a surface-mounted cube in uniform and turbulent streams, J. Fluid Mech., 79, 307335, 1977.

Counihan, J., Hunt, J. C. R., and Jackson, P. S.: Wakes behind twodimensional surface obstacles in turbulent boundary layers, J. Fluid Mech., 64, 529-563, 1974.

Goring, D. G. and Nikora, V. I.: Despiking acoustic Doppler velocimeter data, J. Hydraul. Eng., 128, 117-126, 2002.

Gsänger, S. and Pitteloud, J.: Small wind world report 2014, Tech. rep., World Wind Energy Association, Bonn, 2014.

Iaccarino, G., Ooi, A., Durbin, P. A., and Behnia, M.: Reynolds averaged simulation of unsteady separated flow, Int. J. Heat Fluid Flow, 24, 147-156, 2003.
Karlsson, C. J., Olsson, F., Letalick, D., and Harris, M.: All-fiber multifunction continuous-wave coherent laser radar at 1.55 num for range, speed, vibration, and wind measurements, Appl. Optics, 39, 3716-3726, 2000.

Lenschow, D. H., Mann, J., and Kristensen, L.: How long is long enough when measuring fluxes and other turbulence statistics?, J. Atmos. Ocean. Tech., 11, 661-673, 1994.

Liu, H., Peters, G., and Foken, T.: New equations for sonic temperature variance and buoyancy heat flux with an omnidirectional sonic anemometer, Bound.-Lay. Meteorol., 100, 459-468, 2001.

Mortensen, N. G., Heathfield, D. N., Myllerup, L., Landberg, L., and Rathmann, O.: Getting started with WAsP 9, Tech. Rep. Ris $\varnothing$-I-2571(EN), Risø National Laboratory, Roskilde, Denmark, 2007.

Nägeli, W.: Untersuchungen über die Windverhältnisse im Bereich von Schilfrohrwänden, Mitt. Schweiz. Anst. Forstl. Versuchw., 29, 213-266, 1953.

Nord, M.: Shelter effects of vegetation belts - Results of field measurements, Bound.-Lay. Meteorol., 54, 363-385, 1991.

Peña, A.: Sensing the wind profile, Tech. Rep. Ris $\varnothing-\mathrm{PhD}-45(\mathrm{EN})$, Risø DTU, 2009.

Perera, M. D. A. E. S.: Shelter behind two-dimensional solid and porous fences, J. Wind Eng. Ind. Aerodyn., 8, 93-104, 1981.

Seginer, I.: Windbreak drag calculated from the horizontal velocity field, Bound.-Lay. Meteorol., 3, 87-97, 1972.

Seginer, I.: Atmospheric-stability effect on windbreak shelter and drag, Bound.-Lay. Meteorol., 8, 383-400, 1975.

Sjöholm, M., Angelou, N., Hansen, P., Hansen, K. H., Mikkelsen, T., Haga, S., Silgjerd, J. A., and Starsmore, N.: Two-dimensional rotorcraft downwash flow field measurements by lidar-based wind scanners with agile beam steering, J. Atmos. Ocean. Tech., 31, 930-937, 2014.

Sonnenschein, C. M. and Horrigan, F. A.: Signal-to-noise relationships for coaxial systems that heterodyne backscatter from the atmosphere, Appl. Optics, 10, 1600-1604, 1971.

Sørensen, N. N.: General purpose flow solver applied to flow over hills, Tech. Rep. Ris $\varnothing-\mathrm{R}-827(\mathrm{EN})$, Ris $\varnothing$ National Laboratory, Roskilde, Denmark, 2003.

Stull, R. B.: An introduction to boundary layer meteorology, Kluwer Academic Publishers, Dordrecht, the Netherlands, 1988.

Taylor, P. A. and Salmon, J. R.: A model for the correction of surface wind data for sheltering by upwind obstacles, J. Appl. Meteorol., 32, 1683-1694, 1993.

Wang, H. and Takle, E. S.: On shelter efficiency of shelterbelts in oblique flows, Agr. Forest Meteorol., 81, 95-117, 1996.

Wang, H., Takle, E. S., and Shen, J.: Shelterbelts and windbreaks: Mathematical modeling and computer simulations of turbulent flows, Annu. Rev. Fluid Mech., 33, 549-586, 2001.

Wilson, J. D.: Oblique, stratified winds about a shelter fence. Part I: Measurements, J. Appl. Meteorol., 43, 1149-1167, 2004. 